\title{
Atlas Digital del Plan GAM 2013
}

\author{
Digital Atlas of GAM 2013 Plan
}

\section{Keren Ruiz-Agüero' \\ Casia Soto-Montoya ${ }^{2}$ \\ Tomás Martínez-Baldares ${ }^{3}$}

Fecha de recepción: 04 de febrero del 2014

Fecha de aprobación: 18 de mayo del 2014

Ruiz-Agüero, K; Soto-Montoya, C; Martínez-Baldares, T. Atlas Digital del Plan GAM 2013. Tecnología en Marcha. Vol. 27, N 4, Octubre-Diciembre. Pág I 02- I 12.

I Estudiante del programa de licenciatura de Ingeniería Forestal del Instituto Tecnológico de Costa Rica. Correo electrónico: kerenruiza@gmail.com

2 Licenciada en Ingeniería Forestal. Encargada del Sistema de Información Geográica del Plan GAM 2013. Cartago, Costa Rica. Correo electrónico: cassiaspp@gmail.com. Teléfono: (506)255025। I.

3 Licenciado en Arquitectura y Magister Scientiae en Diseño Urbano. Compañía Nacional de Fuerza y Luz. Profesor de la Escuela de Arquitectura y Urbanismo del Instituto Tecnológico de Costa Rica. Secretario Ejecutivo del Plan GAM 2013. Correo electrónico: tomarbal@gmail.com. Teléfono: (506)25509036. 


\section{Palabras clave}

SIG; diccionario de datos; Plan GAM 20 I3; cartografía; información geográfica; estandarización.

\section{Resumen}

En 2012, en un proceso de colaboración entre la ONU-Hábitat, el Instituto Tecnológico de Costa Rica, el Ministerio de Vivienda y Asentamientos Humanos y el Instituto Nacional de Vivienda y Urbanismo, se oficializó la iniciativa denominada Plan GAM 2013, coordinada por la Secretaría del Plan Nacional de Desarrollo Urbano (SPNDU).

Para consolidar las propuestas y alcanzar los objetivos de este plan fue necesario generar un instrumento de información geográfica capaz de analizar y visualizar los contenidos técnicos del proceso de planificación y ordenamiento territorial. Para ello se utilizó el equipo de software y hardware disponible en la SPNDU y en el Centro Nacional de Alta Tecnología (CeNAT). Además se aplicó una metodología que incluyó los procesos de recopilación y el diagnóstico de la información existente, la creación de un diccionario de datos, la estandarización de los elementos cartográficos, la elaboración de metadatos y la generación de nuevas capas. El resultado de esta metodología fue una base de datos simple, accesible, de fácil manejo y con posibilidades de ser replicada, que se constituyó en un atlas digital con 83 mapas y 172 capas de información geográfica. El uso de este atlas digital permitió analizar de manera eficiente la situación de la Gran Área Metropolitana (GAM), Costa Rica.

\section{Key words}

GIS; data dictionary; cartography; geographic information; standardization.

\section{Abstract}

In 2012 a colaboration process between ONUHabitat, Technological Institute of Costa Rica, the Ministry of Housing and Human Settlements and the National Institute of Housing and Urban Development, was oficialized under he cordination of Secretariat of Urban Development Plan (SPNDU) the initiative called Plan GAM 2013. To consolidate the proposals and objectives to achieve this plan was necessary to generate a GIS tool able to analyze and visualize the technical content of the planning and zoning, to achieve this objective, software and hardware available in the SPNDU and the National Center for High Technology (CeNAT) was used, also a methodology that included the compilation and diagnosis of existing information, the creation of a data dictionary, standardization of cartographic elements, metadata development and the generation of new layers. The result of this approach was a simple database, accessible, easy to use with the potential to replicate, which was incorporated into a digital atlas with 83 maps and I 72 layers of geographic information. The use of this digital atlas allowed an efficient analyze of the situation at the greater metropolitan area in Costa Rica.

\section{Introducción}

El ordenamiento territorial busca maximizar la eficiencia de los procesos de ocupación, aprovechamiento del territorio y sus recursos (Cardona, 200 I). Carvajal (20II) menciona que los planes de ordenamiento territorial permiten observar la visión de territorio que se propone y la legitimación de su práctica a través de actores institucionales. Para lograr esto es necesario optimizar la toma de decisiones en la gestión de una región y construir bases de datos geográficos que se integren a distintos aspectos temáticos y de cartografía que permitan la presentación de resultados (Bosque y García, 200 I; Becerras et al., 2008). Los Sistemas de Información Geográfica (SIG) son una herramienta de gran utilidad en el proceso de planificación territorial.

En términos generales, los SIG abarcan una serie de funciones tales como la actualización, el almacenamiento, la gestión, la presentación y el análisis de datos geoespaciales (Bernhardsen, 2002a). En el ordenamiento del territorio, el principal objetivo de los SIG es apoyar las políticas de administración 
local y regional, de manera que el desarrollo socioeconómico del territorio y la funcionabilidad de los sistemas naturales sean compatibles (Castell et al., 2003)

En Costa Rica, a finales de 2012 se oficializó el proceso Plan GAM 20I3, mediante la reactivación de la Secretaría del Plan Nacional de Desarrollo Urbano (SPNDU) y del Consejo Nacional de Planificación Urbana instaurados por el Decreto 31063. En este proceso se evidenció la necesidad de generar y aplicar una metodología eficiente para la creación de un Atlas Cartográfico Digital que permitiera visualizar y analizar los contenidos técnicos del Plan GAM 2013.

Este artículo presenta la metodología que se utilizó con el objetivo de generar un Atlas Digital de información geográfica para lograr un manejo eficiente de la información en el proceso de elaboración del Plan GAM 2013. Además, el trabajo realizado facilitará la actualización de los futuros planes de ordenamiento territorial.

\section{Materiales y Métodos}

Área de estudio

La Gran Área Metropolitana (GAM) de Costa Rica posee 1780 kilómetros cuadrados comprendidos entre el Valle Central y el Valle del Guarco. La figura I muestra la delimitación de las provincias y cantones de la GAM.

La metodología incluyó las etapas de categorización de la información, conformación del diccionario de datos, estandarización de la información, creación de metadatos, recopilación de la información, postprocesamiento y producción de información geográfica y publicación de mapas.

\section{Categorización de la información}

Al inicio del proyecto se definieron I I categorías (cuadro I) para clasificar las capas de información geográfica y ordenar la información de las seis dimensiones del Plan GAM 2013.

\section{División político administrativa y límites - Plan GAM 2013}

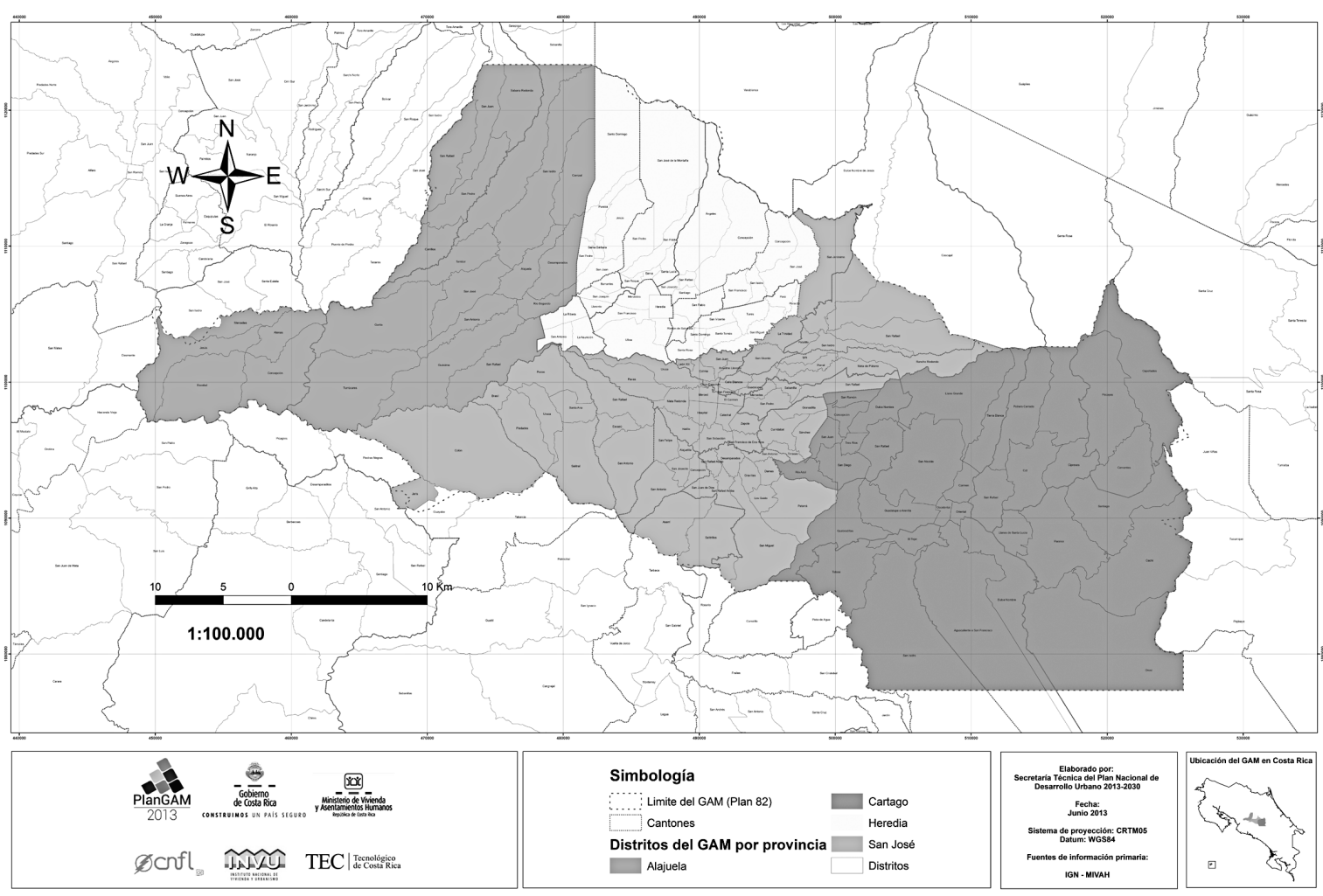

Figura I. División político-administrativa de la GAM. Fuente: Plan GAM 2013 
Cuadro I. Descripción de las categorías de información geográfica.

\begin{tabular}{|l|c|l|}
\hline \multicolumn{1}{|c|}{ Nombre } & Iniciales & \multicolumn{1}{|c|}{ Ejemplos } \\
\hline Información de Referencia & IR & Distritos, Cantones, Provincias \\
\hline Delimitación y Zonificación Regional & DZR & Anillo de contención y borde del GAM. \\
\hline Red Vial Regional & RVR & Plan Nacional de Transporte y anillo de circunvalación. \\
\hline Sistema Integrado de Transporte Público & SITP & Rutas de buses, tren, paradas y estaciones. \\
\hline Áreas Protegidas & AP & $\begin{array}{l}\text { Áreas Silvestres Protegidas, Corredores Biológicos y } \\
\text { propuestas de Corredores Biológicos. }\end{array}$ \\
\hline Sistema Hídrico & SH & $\begin{array}{l}\text { Cuencas, subcuencas, microcuencas, pozos, zonas de } \\
\text { recarga y mantos acuíferos. }\end{array}$ \\
\hline Amenazas & A & $\begin{array}{l}\text { Amenazas de fallas, inundación, erupción volcánica y } \\
\text { deslizamientos. }\end{array}$ \\
\hline Zonas Industriales y Parques Tecnológicos & ZIPT & $\begin{array}{l}\text { Zonas francas e industriales y propuestas de parques } \\
\text { industriales. }\end{array}$ \\
\hline $\begin{array}{l}\text { Vivienda-Equipamientos Colectivos e } \\
\text { Infraestructura de Redes }\end{array}$ & VECIR & $\begin{array}{l}\text { EBAlS, territorios sociales, educación, cobertura de } \\
\text { alcantarillado, radiobases y telefonía fija. }\end{array}$ \\
\hline Sistema Ecológico & SE & $\begin{array}{l}\text { Índices de Fragilidad Ambiental, Unidades del Paisaje, } \\
\text { cobertura del suelo, relieve y condiciones bioclimáticas. }\end{array}$ \\
\hline Zonas de Control Especial & $\begin{array}{l}\text { Reserva indígena Quitirrisí, Zonas Protectoras y conos } \\
\text { de aproximación de los aeropuertos. }\end{array}$ \\
\hline
\end{tabular}

\section{Conformación del diccionario de datos}

En la memoria C:l del computador se creó un sistema de I I carpetas correspondientes a las categorías de información geográfica. Cada una de estas se dividió en carpetas con un número consecutivo según la cantidad de capas de cada clase, y estas a su vez se subdvidieron en tres carpetas llamadas: FUENTE, MAPA y SHAPE, como se muestra en la figura 2; cada una de estas carpetas almacenó un tipo diferente de información sobre la capa.

Además de las I I carpetas, se creó una más con el nombre de METADATOS, que almacenó un archivo Excel que se organizó en dos bases de datos:

Diccionario de capas geográficas en formato shape (figura 3). En este se vinculó cada capa con la categoría y número de carpeta en la que se almacenó, se indicó el nombre (final y actual), el sistema de proyección final, el origen, las modificaciones y el nombre del responsable de la capa.

El diccionario de mapas (figura 4). Este se estructuró por dimensión y se creó un enlace con los mapas contenidos en el documento del Plan GAM 2013.
Este diccionario también enlazó cada mapa con la categoría y número de carpeta en la que se almacenó.

En el ejemplo de las figuras 3 y 4 se puede observar el campo de "Identificador", el cual se caracterizó por ser un código único, ya que el número consecutivo de la capa (ID_CAPA) y del mapa (ID_MAPA) es irrepetible dentro de una categoría. En la figura 5 se puede observar la estructura del identificador.

En la homogeneización del nombre de capas (figura 6) y mapas (figura 7) se utilizó una estructura sencilla que incluyó, entre otros aspectos, la fecha de creación de los elementos.

Para el manejo de la información y evitar problemas como la duplicación de datos y confusión de capas, se tomaron en cuenta las siguientes recomendaciones:

- A las carpetas dentro de una categoría se les asignó una numeración consecutiva que se inició en 0 I.

- Las capas tuvieron el carácter de exclusivas de cada categoría, es decir, no se repitieron en ninguna otra carpeta. 


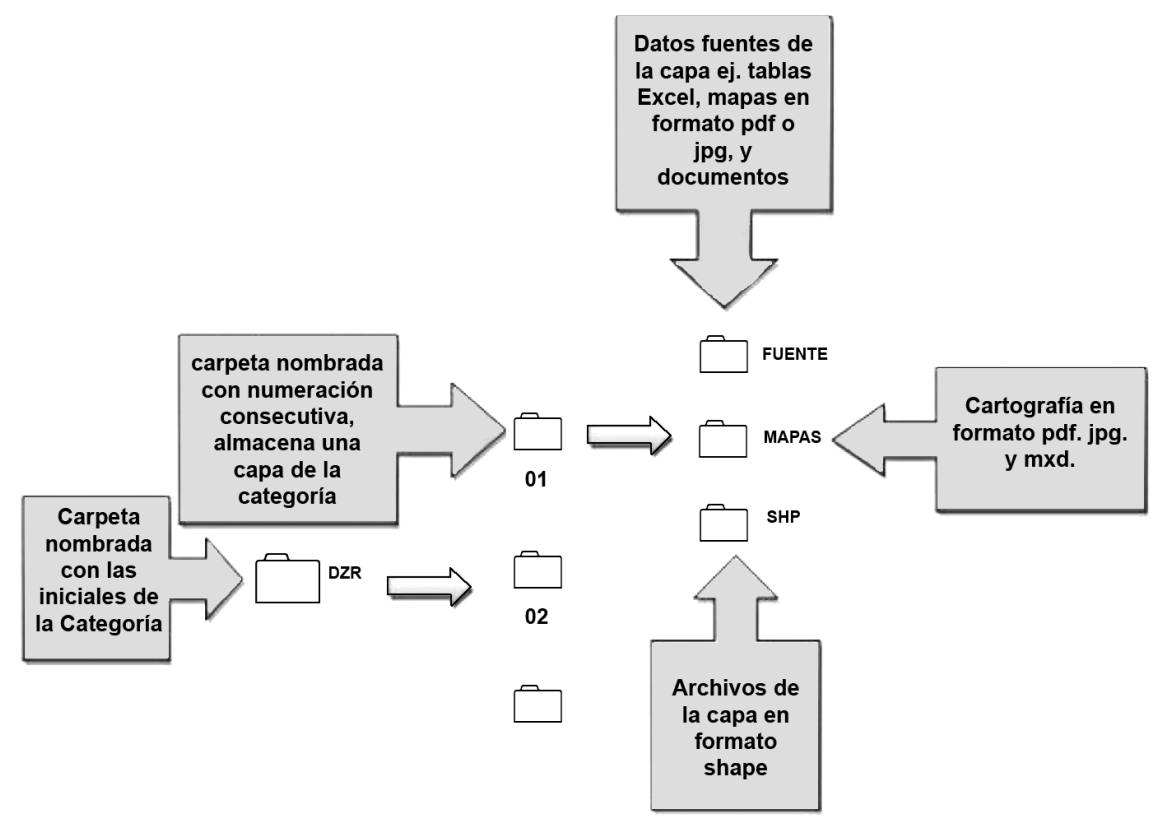

Figura 2. Modelo del diseño del diccionario de datos.

\begin{tabular}{|c|c|c|c|c|c|c|c|c|c|c|}
\hline $\begin{array}{l}\text { ID de la } \\
\text { categoría }\end{array}$ & $\begin{array}{c}\text { ID de la } \\
\text { Capa }\end{array}$ & Identificador & Clasificación & Nombre final & $\begin{array}{c}\text { Nombre } \\
\text { actual }\end{array}$ & Tipo & $\begin{array}{l}\text { Sistema de } \\
\text { proyección }\end{array}$ & Origen & Modificaciones & $\begin{array}{l}\text { Nombre de } \\
\text { Responsable }\end{array}$ \\
\hline A & 01 & A_Ol & Amenazas & $\begin{array}{c}\text { Fallas_Clasificadas_- } \\
\text { GAM_2013_10_10 } \\
\text { shp }\end{array}$ & $\begin{array}{l}\text { fallas_ } \\
\text { Clasificadas } \\
\text { _2013.shp }\end{array}$ & shp & CRTM05 & $\begin{array}{l}\text { C:IGAM2013_C } \\
\text { NEISismologíal } \\
\text { fallas_Clasifica } \\
\text { das_2013.shp }\end{array}$ & $\begin{array}{c}\text { Cambio de } \\
\text { nombre }\end{array}$ & CNE \\
\hline
\end{tabular}

Figura 3. Formato del registro de capas del Atlas Digital del Plan GAM 2013.

\begin{tabular}{|c|c|c|c|c|c|c|c|c|}
\hline Dimensión & $\begin{array}{c}\mathrm{N}^{0} \text { de } \\
\text { figura en el } \\
\text { documento }\end{array}$ & $\begin{array}{l}\text { ID de la } \\
\text { categoría }\end{array}$ & $\begin{array}{c}\text { ID de la } \\
\text { carpeta (ID } \\
\text { de la capa) }\end{array}$ & $\begin{array}{l}\text { ID del } \\
\text { mapa }\end{array}$ & Identificador & Clasificación & Nombre & Contenido \\
\hline DA & DA-5.3.3-8 & A & 01 & 01 & A_Ol_Ol & Amenaza & $\begin{array}{l}\text { A_0I_0I_Amenaza_Fallas_ } \\
\text { Geologicas_2013_I0 }\end{array}$ & $\begin{array}{c}\text { DZR_0I/ } \\
\text { DZR_I2/IR_02/ } \\
\text { IR_03/IR_05/ } \\
\text { RAV_0| }\end{array}$ \\
\hline
\end{tabular}

Figura 4. Formato del registro de mapas del Atlas Digital del Plan GAM 2013.
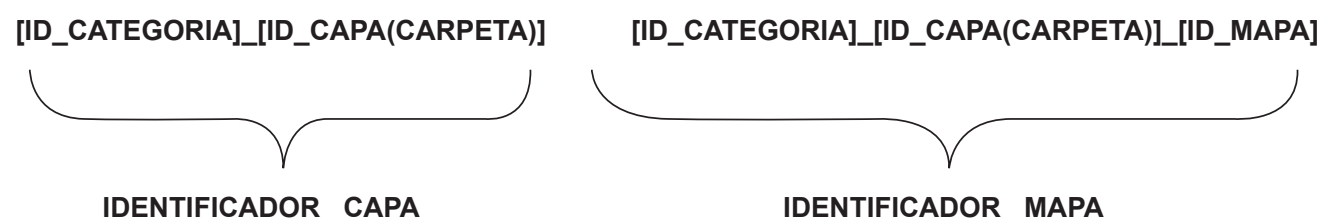

Figura 5. Estructura del elemento "Identificador" del diccionario de datos. 


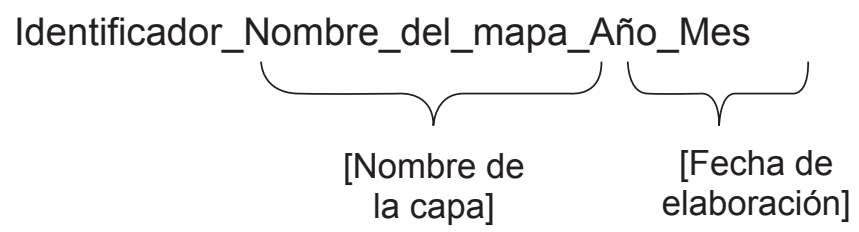

Figura 6. Estructura del nombramiento de las capas en el diccionario de datos.

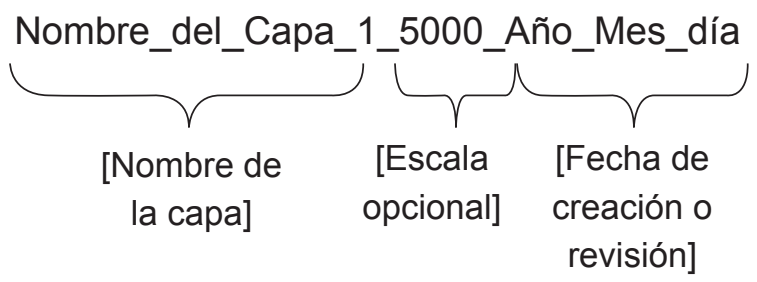

Figura 7. Estructura del nombramiento de mapas en el diccionario de datos.

- La carpeta "MAPA" puede no contener ningún mapa o tener más de uno.

- La información que se localizó en la carpeta "FUENTE" estuvo sujeta a la disponibilidad de documentación relacionada con el origen de la capa.

- La carpeta "SHP" contiene una única capa en formato shapefile y siempre es la última versión de la capa.

- Las capas temporales se crearon en una carpeta de prueba.

- El identificador (ID) de la categoría y carpeta pudo haberse repetido, pero el número del mapa nunca se repitió.

- A los mapas se les asignó el identificador de la capa principal, aunque estuvieran conformados por varias capas de referencia.

\section{Recopilación de la información}

Se recopiló la información del PRUGAM, en el Ministerio de Vivienda y Asentamientos Humanos (MIVAH), y del POTGAM, en el Instituto Nacional de Vivienda e Urbanismo (INVU). Además, se incorporó información proveniente de instituciones como el Instituto Tecnológico de Costa Rica (TEC) (proyecto Atlas Digital de Costa Rica 2008), el Sistema Nacional de Áreas de Conservación
(SINAC), el Instituto Costarricense de Acueductos y Alcantarillados (AyA), el Servicio Nacional de Aguas Subterráneas, Riego y Avenamiento (SENARA), los municipios, el Instituto Nacional de Estadísticas y Censos (INEC), la Compañía Nacional de Fuerza y Luz (CNFL), el Ministerio de Ciencia y Tecnología (MICIT) y la Comisión Nacional de Prevención de Riesgos y Atención de Emergencias (CNE).

\section{Postprocesamiento y producción de información geográfica}

Se realizó un diagnóstico con el software ArcGIS I0.I, en el cual se revisó el sistema de proyección, posición geográfica y el contenido de la tabla de atributos. En el postprocesamiento fue necesario proyectar a CRTM05 (sistema oficial para Costa Rica), añadir información complementaria a la tabla de atributos y aplicar operaciones de geoprocesamiento. La producción de nuevas capas se realizó a partir de información alfanumérica e imágenes georreferenciadas. Los procesos que requirieron una alta capacidad de procesamiento en esta etapa se efectuaron con el equipo que facilitó el Centro Nacional de Alta Tecnología (CeNAT) en sus instalaciones.

A las capas se les realizaron modelos cartográficos en Word y metadatos que se elaboraron en Excel, basándose en la plantilla que creó el Instituto Geográfico Nacional (IGN) para la información 
vectorial, la cual utilizó como base la norma ISO 19| 15:2003 Geographic Information Metadata, la norma ISO 19115:2003 Geographic Information Services y el Perfil Latinoamericano de Metadatos (LAMP) (Registro Nacional, s. f.).

\section{Publicación de mapas}

Se estableció una plantilla estándar para todos los mapas (figura 8), y se añadió en la parte superior izquierda una marca de agua con el número de figura de los mapas que aparecen en el documento oficial del Plan GAM 2013.

\section{Resultados y Análisis}

El producto de la metodología del Atlas Digital para el Plan GAM 2013 se constituyó en un diccionario de datos actualizado, consolidado y ordenado, que facilitó la incorporación de nueva información. Otros proyectos similares, como el SITXELL (Sistema d' Informació Territorial de la Xarxa d' Espais Lliures de la provincia de Barcelona), también se basaron en crear una estructura de módulos o categorías temáticas que cubren los diversos aspectos que intervienen en el ordenamiento territorial (Castell et al., 2003) y cuyo principal objetivo es describir la estructura de los datos (Bernhardsen, 2002b).
La utilización del diccionario de datos agilizó los procesos, lo cual recompensó algunas dificultades que se presentaron, como los atrasos en el envío de información geográfica por parte de las instituciones, las capas desplazadas o con errores de traslape, las capas definidas en sistemas de proyección no reconocidos u oficiales y los mapas cartográficos ilegibles.

El Atlas Digital del Plan GAM incluyó un total de 83 mapas y 172 capas. En la figura 9 se muestra el porcentaje de mapas distribuido por categoría.

La categoría que presentó mayor cantidad de mapas es la de Vivienda y Equipamiento Colectivo e Infraestructura de Redes (VECIR), debido a que en esta se localizaron mapas para dos dimensiones: Equipamiento Social e Infraestructura de Redes. Algunos de estos mapas son Territorios Sociales (figura 10), Equipamiento Social-EBAIS, Condición de Vivienda, Cobertura de Alcantarillado, Cobertura de Radiobases Instaladas y por Instalar y Distribución de Antenas de Celular (ICE).

Las clases con menor porcentaje de mapas fueron Sistema Integrado de Transporte Público (SITP) y Zonas Industriales-Parques Tecnológicos (ZIPT); en estas categorías se registró un mapa que integró la mayoría de las capas (figura II).

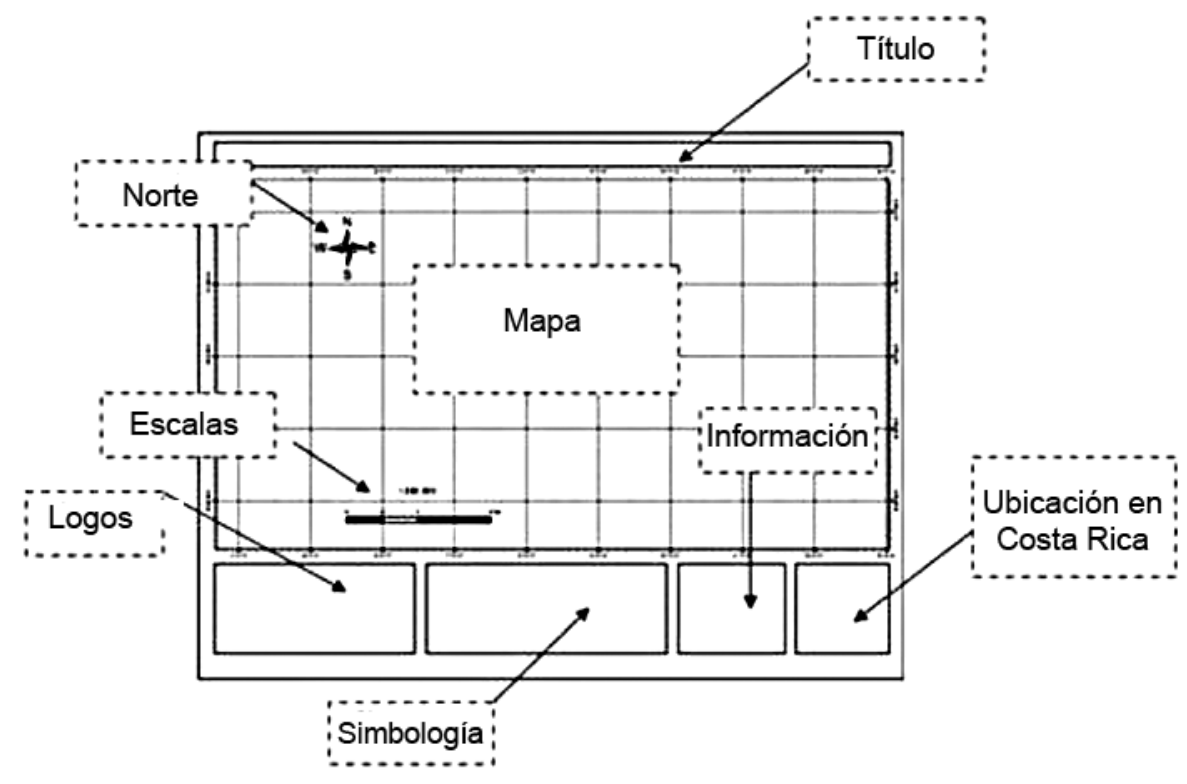

Figura 8. Plantilla estándar de mapas del Atlas Digital del Plan GAM 2013. 


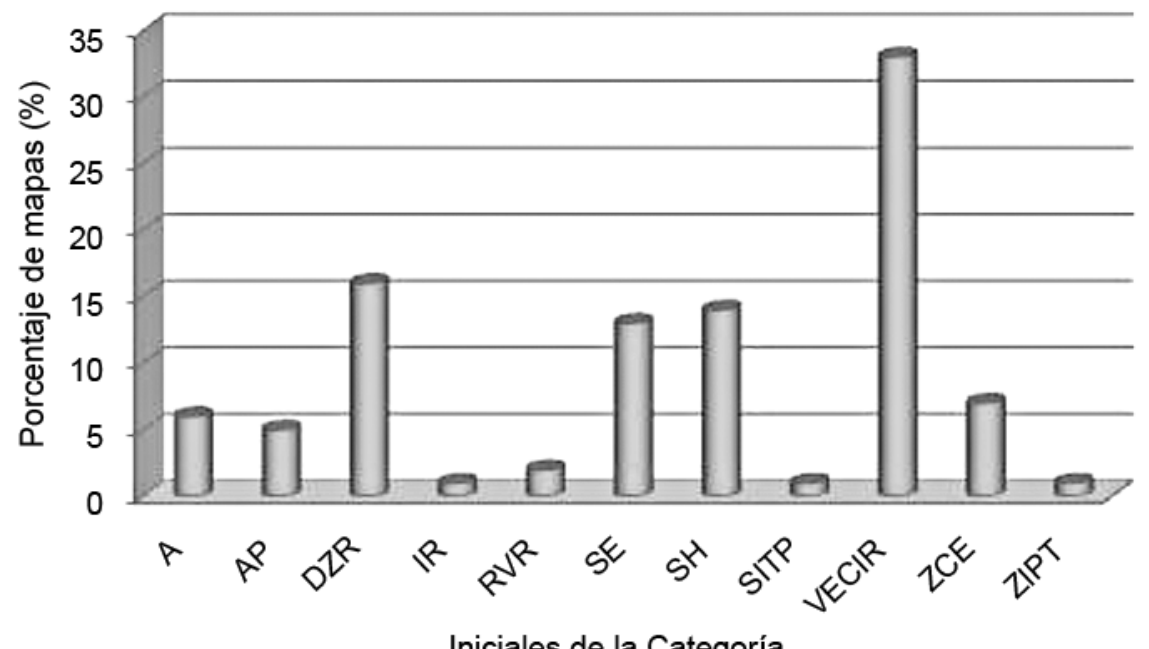

Iniciales de la Categoría

Figura 9. Gráfico del porcentaje de mapas por categoría.

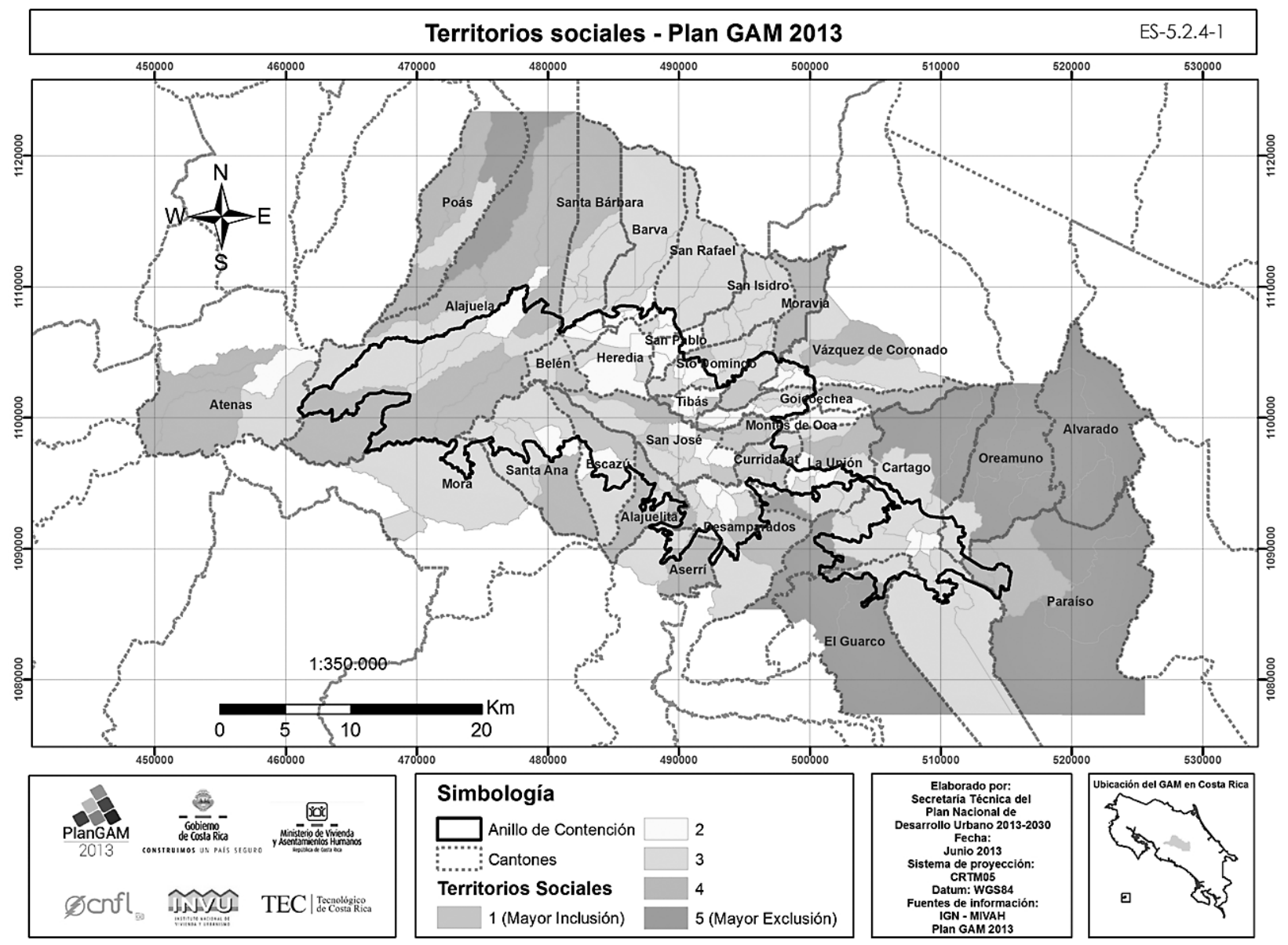

Figura 10. Territorios Sociales, Atlas Digital del Plan GAM 20I3. Fuente: Plan GAM 2013. 


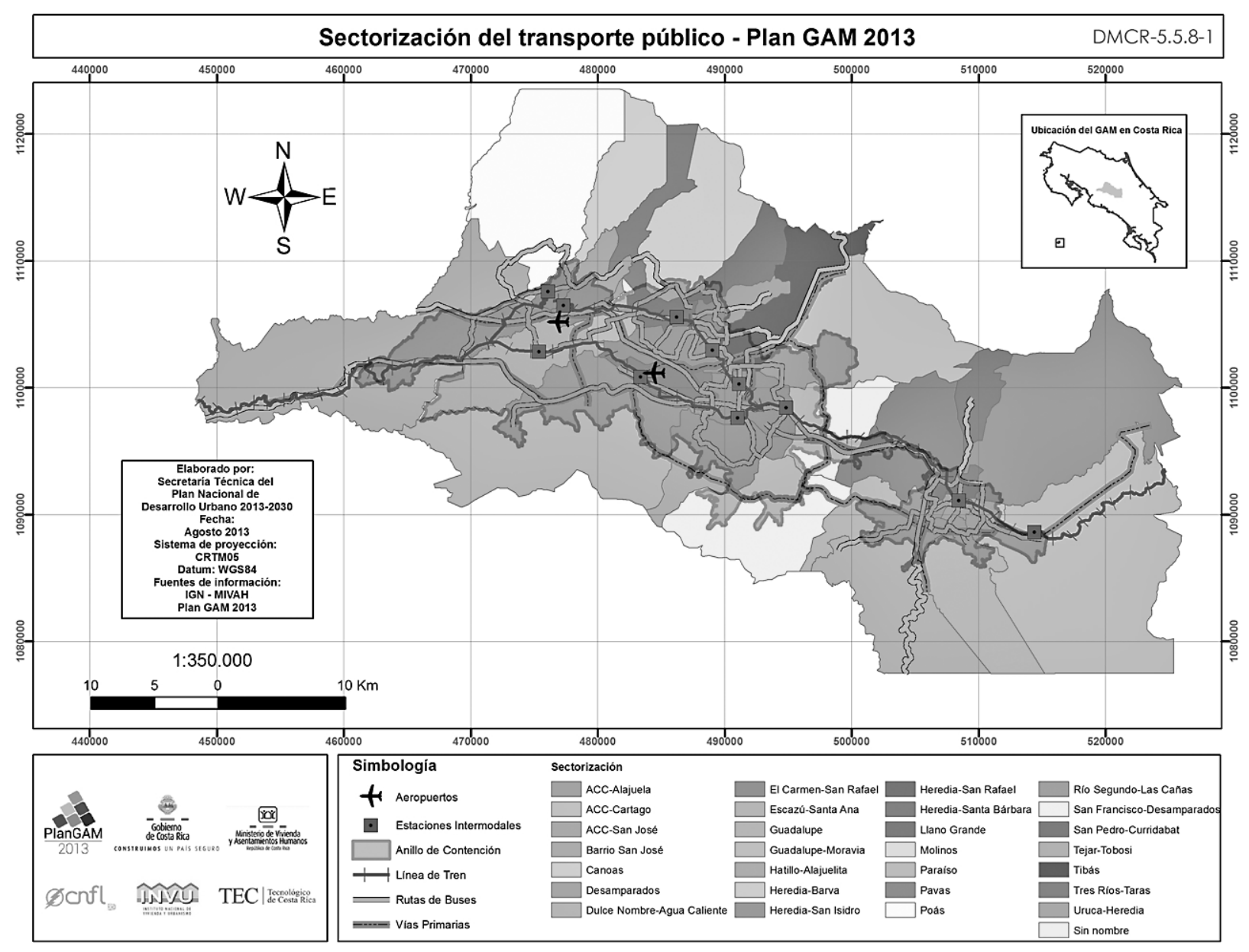

Figura I I. Integración de la información en el mapa de Sectorización del Transporte Público. Fuente: Plan GAM 20 I 3.

La dimensión ambiental abarcó una complejidad de temas e incorporó mapas de diferentes categorías. Además, presentó el mayor porcentaje de mapas, entre los cuales destacan Amenaza de Fallas Geológicas, Capacidad de Recarga Hídrica (figura 12) y Zonas de Vida.

En la figura 13 se observa la distribución de porcentaje de capas por categoría.

Como se muestra en la figura 13, la mayoría de las capas se concentraron en las categorías DZR,VECIR y SE, debido a la generalidad de estas clases. Otras categorías, como Amenazas (A) y Zonas Industriales y Parques Tecnológicos (ZIPT), presentaron menor cantidad de capas debido a que su especificidad es mayor.

En relación con la generación o procesamiento de la información geográfica, los modelos cartográficos sirvieron para respaldar el origen de las capas, lo cual es importante para garantizar un proceso objetivo, transparente y replicable.

La generación de metadatos estandarizados es fundamental para permitir el intercambio de información geográfica y mantener un control de los datos en el tiempo.

\section{Conclusiones}

La revisión de la información permitió seleccionar las capas geográficas confiables y actualizadas. Además, solventó los vacíos de datos y sirvió de apoyo para la creación de nuevas capas.

El uso de normas de estandarización en el diccionario, el empleo de información actual, la construcción de modelos cartográficos y la creación de metadatos 


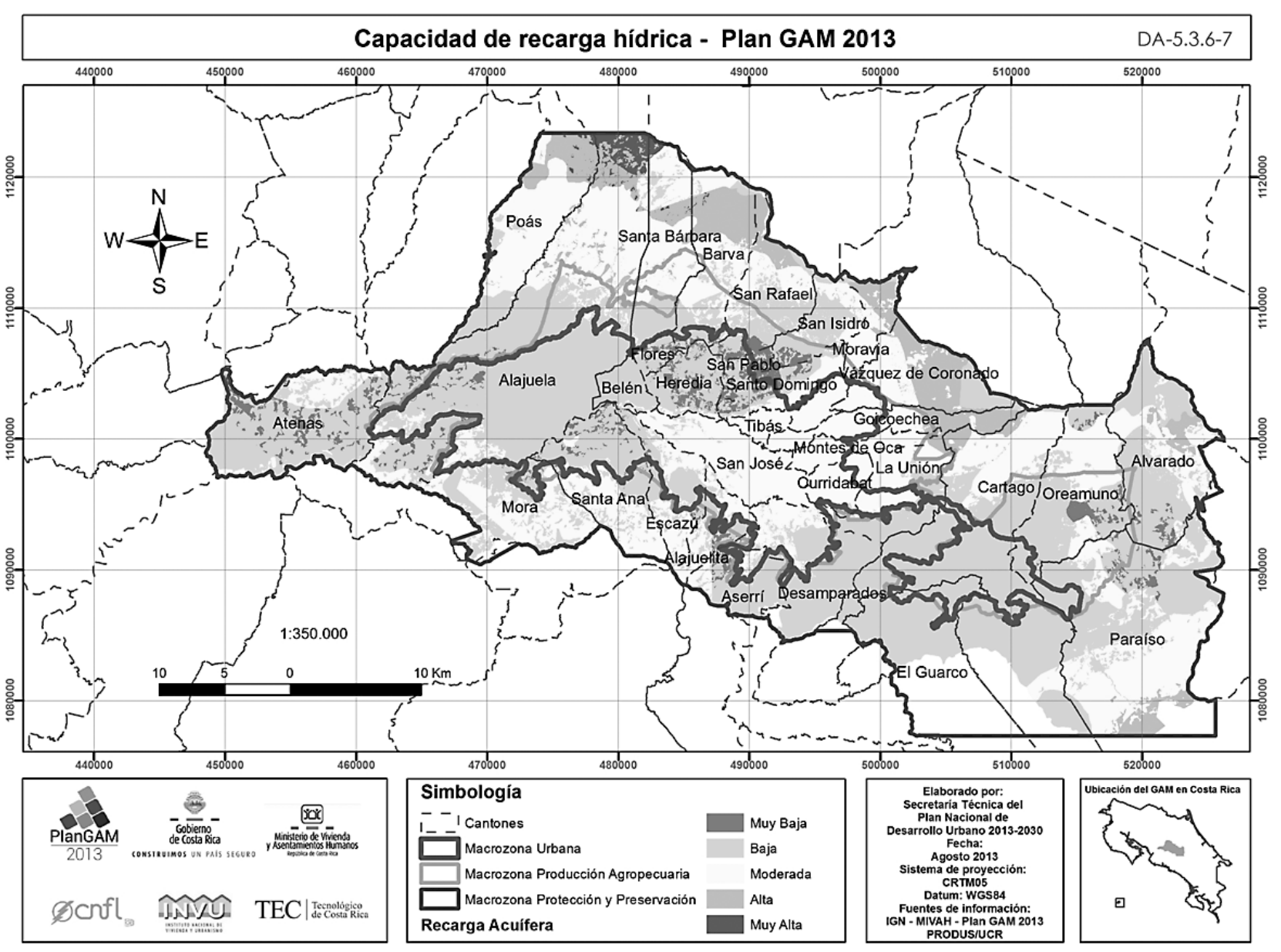

Figura 12. Capacidad de Recarga Hídrica. Fuente: Plan GAM 2013.

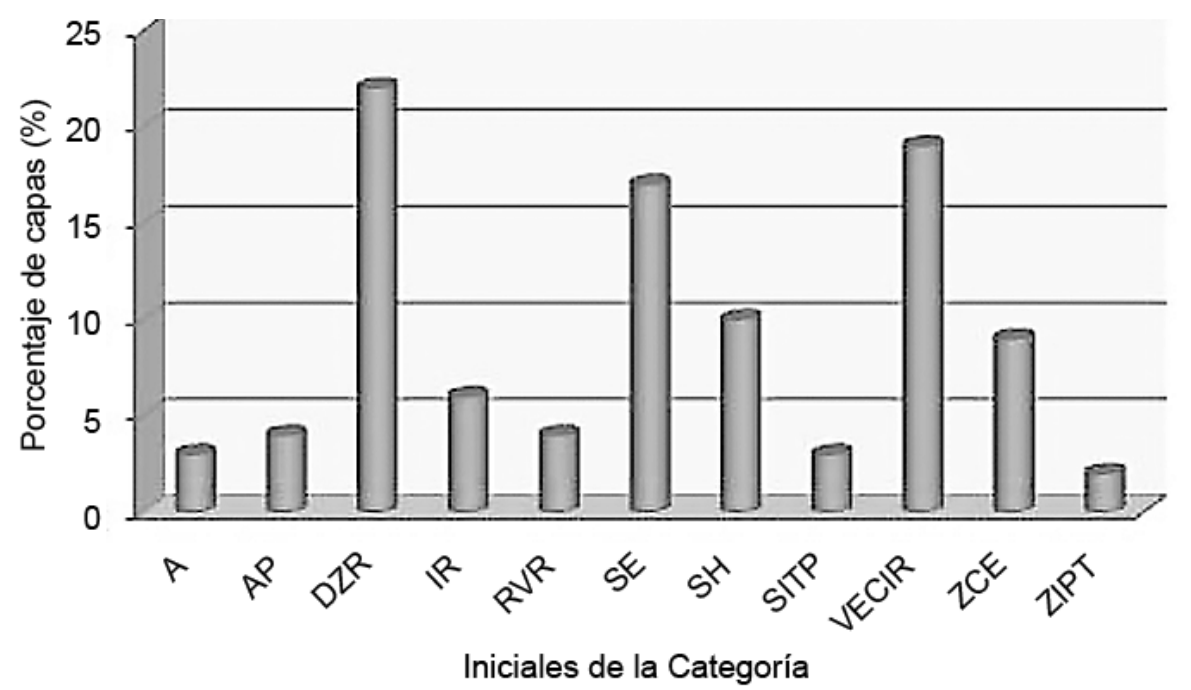

Figura 13. Gráfico del porcentaje de capas por categoría. 
bajo un estándar fortaleció la calidad del Atlas Digital del Plan GAM 2013.

A pesar de las dificultades que se presentaron en el Atlas Digital, la aplicación de una metodología basada en un diccionario de datos agilizó los procesos de búsqueda, incorporación y actualización de capas y mapas. Además, creó una base de datos simple, fácil de manejar y de replicar.

El Atlas Digital amplió las posibilidades de desarrollar instrumentos complementarios como, por ejemplo, geoportales y herramientas interactivas; esto permitiría la conectividad entre municipios, simplificaría los trámites y facilitaría la actualización a nivel regional.

El Atlas Digital del Plan GAM 2013 se consolidó en una herramienta tecnológica, ordenada, eficaz y objetiva que facilitó el análisis de la situación de la GAM.

\section{Bibliografía}

Becerras, E., Martínez, R., Pineda, E., Barreto, B., Hernández, N., Vidal, M., Rodríguez, I., Acosta., F. \& Cruz, A. (2008). Ordenamiento territorial y sistema de información en la agricultura cañera en Villa Clara. Centro Agrícola, 35(4), 47-5 I.

Bernhardsen, T. (2002a). Standards and Geographical Infraestructure. En Geographic Information Systems (pp. 377-40 I). New York: John Wiley \& Sons.
Bernhardsen, T. (2002b). Geographical Information Systems and Graphical Information. En Geographic Information Systems (pp. 377-40I). New York: John Wiley \& Sons.

Bosque, J. \& García, R. C. (2000). El uso de los sistemas de información geográfica en la planificación territorial. Anales de Geografía de la Universidad Complutense, 20, 49-67.

Cardona, A. H. (200I). Reflexiones para un Futuro en el Ordenamiento Territorial y la Planificación de las Regiones Colombianas: Influencia del Medio Ambiente Global. Revista Jurídica Piélagus, 2, 53-57.

Carvajal Sánchez, N. (20 I I). La dinámica del ordenamiento territorial en Colombia: contraste de dinámicas urbano-rurales en la región metropolitana de Bogotá. (Tesis Doctoral). Universidad de Montreal, Ottawa, CA.

Castell, C., Beltran, A. \& Margall, M. (2003). El Sistema d' Informació Territorial de la Xarxa d'Espais Lliures (SITXELL) de la provincia de Barcelona. En Diputación de Barcelona (Ed.), El territorio como sistema (pp. I59179). Barcelona: Institut d'Edicions de la Diputació de Barcelona.

Instituto Panamericano de Geografía e Historia. (201 I). Guía de Normas Edición en español Comité ISO/TC 2II Información Geográfica/Geomática. Distrito Federal, MX. Obtenido de http://www.isotc2II.org/Outreach/ISO_ TC_2II_Standards_Guide_Spanish.pdf

Registro Nacional. (20।3). Borrador del perfil oficial de metadatos para datos geoespaciales de Costa Rica. San José: Instituto Geográfico Nacional. 